\title{
Integrated analysis of chromosome copy number variation and gene expression in cervical carcinoma
}

\author{
Deng Yan ${ }^{1,2, *}$, Song $\mathbf{Y i}^{1, *}$, Wang Chi Chiu ${ }^{1,2}$, Liu Gui Qin ${ }^{3}$, Wong Hoi Kin ${ }^{1}$, Chung Tony \\ Kwok Hung ${ }^{1}$, Han Linxiao ${ }^{4}$, Choy Kwong Wai ${ }^{1,2}$, Sui $\mathrm{Yi}^{5}$, Yang $\mathrm{Tao}^{6}$ and Tang $\mathrm{Tao}^{1,2}$ \\ ${ }^{1}$ Department of Obstetrics \& Gynaecology, The Chinese University of Hong Kong, Hong Kong, China \\ ${ }^{2}$ CUHK Shenzhen Research Institute, Shenzhen, China \\ ${ }^{3}$ Shenzhen Laboratory of Ophthalmology, Shenzhen Eye Hospital, Affiliated Shenzhen Eye Hospital of Shenzhen University, \\ Shenzhen, China \\ ${ }^{4}$ Dongguan Third People's Hospital, Dongguan, China \\ ${ }^{5}$ Department of Nutrition, The First Affiliated Hospital of Sun Yat-sen University, Guangzhou, China \\ ${ }^{6}$ Center for Medical Research and Innovation, Shanghai Pudong Hospital, Fudan University Pudong Medical Center, Shanghai, China \\ *These authors have contributed equally to this work \\ Correspondence to: Tang Tao, email: tangtao@cuhk.edu.hk \\ Yang Tao, email: mryangtao2005@yahoo.com \\ Sui Yi, email: suiyisy@hotmail.com
}

Keywords: cervical cancer; chromosome copy number variation; gene expression; cluster analysis; cell cycle pathways

Received: April 19, $2017 \quad$ Accepted: September 21, 2017 Published: November 11, 2017

Copyright: Yan et al. This is an open-access article distributed under the terms of the Creative Commons Attribution License 3.0 (CC BY

3.0), which permits unrestricted use, distribution, and reproduction in any medium, provided the original author and source are credited.

\section{ABSTRACT}

Objective: This study was conducted to explore chromosomal copy number variations (CNV) and transcript expression and to examine pathways in cervical pathogenesis using genome-wide high resolution microarrays.

Methods: Genome-wide chromosomal CNVs were investigated in 6 cervical cancer cell lines by Human Genome CGH Microarray Kit (4x44K). Gene expression profiles in cervical cancer cell lines, primary cervical carcinoma and normal cervical epithelium tissues were also studied using the Whole Human Genome Microarray Kit (4x44K).

Results: Fifty common chromosomal CNVs were identified in the cervical cancer cell lines. Correlation analysis revealed that gene up-regulation or down-regulation is significantly correlated with genomic amplification $(P=0.009)$ or deletion $(P=0.006)$ events. Expression profiles were identified through cluster analysis. Gene annotation analysis pinpointed cell cycle pathways was significantly $(P=1.15 \mathrm{E}-08)$ affected in cervical cancer. Common CNVs were associated with cervical cancer.

Conclusion: Chromosomal CNVs may contribute to their transcript expression in cervical cancer.

\section{INTRODUCTION}

Although the most important etiological agent in cervical cancer is human papillomavirus (HPV) infection, only a small proportion of infected women developed cervical cancer. HPV infection alone is insufficient to induce malignant changes. Copy number variation (CNV) is a very common phenomenon in cervical cancer and may be important in its pathogenesis [1]. Comparative genomic hybridization (CGH) studies for cervical cancer progression have shown that chromosome $3 \mathrm{q}$ gain was associated with the transition from pre-invasive to invasive cervical carcinoma. Subsequently, array-based CGH $(\mathrm{aCGH})$, where arrays of genomic sequences replaced metaphase chromosomes as hybridization targets, was established. More detailed and precise genomic variations had been found in cervical cancer by using aGCH [2]. Lando et al. reported several potential driver genes for cervical carcinogenesis aCGH [3]. However, a recent study reported a limited correlation between chromosomal CNV 
and gene expression by single nucleotide polymorphisms array platform [4].

In this study, a high resolution Human Genome CGH Microarray Kit was used to detect genome-wide chromosomal CNV in 6 cervical cancer cell lines. We also performed gene expression studies in the cervical cancer cell lines, cervical carcinoma tissues and normal cervical epithelia by using Whole Human Genome Microarray Kit. Using appropriate bioinformatics software, we identified several chromosomal CNV regions and aberrantly expressed genes in cervical cancer. Statistical analysis and gene annotation analysis were also performed for the array data.

\section{RESULTS}

\section{Fifty common chromosomal CNVs were identified in cervical cancer cell lines}

Using aCGH at a genome-wide resolution of 250 $\mathrm{kb}$, a total of 50 common chromosomal CNV regions were identified, ranging from $0.5 \mathrm{Mb}$ to $80 \mathrm{Mb}$. Of these, 21 common amplification regions (including 13 significant amplification regions) and 29 deletion regions (including 2 significant deletion regions) were identified (Table 1).

Eleven of these ( 5 amplification regions and 6 deletion regions) have not been previously described in cervical cancer (Table 1). A total of 3514 genes were identified, and many tumor related genes, such as $A B L 1$, BCL3, CDH1, CDKN1C, EPHA3, ERBB4, FOSL1, JUNB, MLH1, MYB, p53, RB1, ROS1, SKI, TGFBR1 and THRB, were located in these 50 chromosomal CNV regions.

\section{Chromosomal CNVs could contribute to their transcript expression in cervical cancer}

To evaluate if there is any association between chromosomal CNVs and gene expression changes in cervical cancer, we analyzed the gene expression profiles of the cervical cancer cell lines and normal cervical epithelium samples. $17.52 \%$ of transcripts $(7,211$ out of 41,152 ) exhibited a 2 -fold over-expression and $9.02 \%$ of transcripts $(3,712$ out of 41,152$)$ displayed a 2 -fold downregulation in 6 cervical cancer cell lines compared with 3 normal cervical epithelium tissues. Within the 21 common genomic amplification regions, $27.94 \%$ of the transcripts (772 out of 2,794) showed 2-fold over-expression. In the 13 significant amplification regions, the percentage was $29.56 \%$ (459 out of 1,553). In the 29 deletion regions, $10.46 \%$ (287 out of 2744 ) revealed 2-fold down-regulation. In the 2 significant deletion regions, the percentage was $11.67 \%$ ( 7 out of 60 ) (Figure $1 \mathrm{~A}$ and $1 \mathrm{~B}$ ). Statistical analysis showed that gene up-regulation or down-regulation was significantly correlated with genomic amplification ( $\mathrm{P}$ $<0.01$, Spearsman correlation test) or deletion $(\mathrm{P}<0.01)$ events. Thus, chromosomal CNVs can contribute to their transcript expression in cervical cancer. Two tumor related genes, $A B L 1$ and $p 53$, which were located in the genomic amplification regions, were found to be over-expressed by at least 2-fold in cervical cancer.

\section{Profiles differed between cervical cancer cell lines, primary cervical carcinoma and normal cervical epithelium tissues}

Expression profiles of transcripts across 6 different cervical cancer cell lines and 2 cervical carcinoma tissues and 3 normal, age-matched, cervical epithelium samples were analyzed using a hierarchical clustering algorithm (unsupervised K-means clustering). Cervical cancer cell lines, cervical carcinoma and normal cervical epithelium were divided two main groups (Figure 2A): cervical cancer cell lines for one group, and clinical cervical carcinoma and normal cervical epithelium for the other group. Interestingly, when gene tree clustering analysis was used to analyze genes with aberrant expression within the 50 common chromosome $\mathrm{CNV}$ regions and 15 significant chromosome $\mathrm{CNV}$ regions, similar results were obtained (Figure $2 \mathrm{~B}$ and $2 \mathrm{C}$ )

\section{Gene ontology analysis for aberrantly expressed genes}

By using a volcano plot in GeneSpring, 9,446 transcripts $(27.4 \%)$ were found to be changed over two fold in the "Cancer group" (including 6 cervical cancer cell lines and 2 clinical cervical carcinomas) compared with the "Normal group" (including 3 cervical epithelium tissues). Among these genes, 6,001 transcripts were upregulated by over 2 fold, and 3,445 transcripts were downregulated by over 2 fold.

\section{Pathway analysis showed that cell cycle pathways, cell communication pathways and DNA polymerase pathways were significantly affected pathways in cervical cancer}

To further investigate the biological significance of these aberrantly expressed genes, pathway analysis was performed. The analysis results showed that cycle cycle pathways $(P=1.15 \mathrm{e}-8)$, cell communication pathways $(P=1.15 \mathrm{e}-8)$ and DNA polymerase pathways $(P=1.15 \mathrm{e}-8)$ were significantly were affected in the cervical cancer (Table 2). Pathway analysis for the 446 differentially expressed transcripts in the 15 significant chromosomal CNV regions also showed that the cell cycle pathway involved the highest number of transcripts (including ABL1, DUSP9, E2F4, TP53, PKMYT1 and PPP1CA) (Figure 3), and the $P$ value is 0.00062 (Table 3 ).

\section{DISCUSSION}

In our genome-wide CNV analysis, we identified 50 frequently altered genomic regions (ranging from 0.5 
Table 1: Physical location of the chromosome CNV regions identified by array CGH in cervical cancer cell lines

\begin{tabular}{|c|c|c|c|c|c|c|c|}
\hline Region & $\begin{array}{l}\text { Region } \\
\text { length }\end{array}$ & $\begin{array}{l}\text { Cytoband } \\
\text { location }\end{array}$ & Event & Genes & Frequency $\%$ & P-value & Reference \\
\hline chr1:10001-3752828 & $3,742,828$ & p36.33 - p36.32 & Gain & 70 & 83.33333333 & 0.003 & $\begin{array}{c}\text { Gopeshwar Narayan, et al., 2007; Connie } \\
\text { P. Matthews, et al., 2000; Y.W. CHOI, } \\
\text { et al., } 2007\end{array}$ \\
\hline chr2:75161025-85137285 & $9,976,261$ & p12-p11.2 & Loss & 17 & 83.33333333 & 0.006 & Y.W. CHOI", et al., 2007 \\
\hline $\begin{array}{l}\operatorname{chr} 2: 137,395,646- \\
170,227,851\end{array}$ & $32,832,206$ & q21.3 - q31.1 & Loss & 84 & 100 & $>0.05$ & $\begin{array}{l}\text { F.Y. Huang et al.2005; Y.W. CHOI, et al., } \\
2007\end{array}$ \\
\hline $\begin{array}{l}\text { chr2:178,374,598- } \\
197,908,813\end{array}$ & $19,534,216$ & q31.2 - q33.1 & Loss & 67 & 83.33333333 & $>0.05$ & $\begin{array}{l}\text { F.Y. Huang et al.2005; Y.W. CHOI, et al., } \\
2007\end{array}$ \\
\hline $\begin{array}{l}\operatorname{chr} 2: 209,391,516- \\
216,053,786\end{array}$ & $6,662,271$ & q34 - q35 & Loss & 15 & 83.33333333 & $>0.05$ & $\begin{array}{l}\text { Lockwood WW, et al., 2007; F.Y. Huang } \\
\text { et al.2005; G Ng, et al., } 2007\end{array}$ \\
\hline $\operatorname{chr} 3: 60,001-8,582,632$ & $8,522,632$ & $\mathrm{p} 26.3-\mathrm{p} 25.3$ & Loss & 15 & 83.33333333 & $>0.05$ & $\begin{array}{c}\text { F.Y. Huang et al.2005; Connie P. } \\
\text { Matthews, et al., 2000; Y.W. CHOI, et } \\
\text { al., } 2007\end{array}$ \\
\hline $\operatorname{chr} 3: 16,297,340-37,460,259$ & $21,162,920$ & $\mathrm{p} 25.1-\mathrm{p} 22.2$ & Loss & 60 & 83.33333333 & $>0.05$ & $\begin{array}{l}\text { Lockwood WW, et al., 2007; F.Y. Huang } \\
\text { et al.2005; Connie P. Matthews, et al., } \\
2000\end{array}$ \\
\hline $\operatorname{chr} 3: 58,690,297-90,181,487$ & $31,491,191$ & $\mathrm{p} 14.2-\mathrm{p} 11.1$ & Loss & 52 & 83.33333333 & 0.028 & $\begin{array}{c}\text { Lockwood WW, et al., 2007; F.Y. Huang } \\
\text { et al.2005; Connie P. Matthews, et al., } \\
\text { 2000; Y.W. CHOI, et al., 2007; G Ng, et } \\
\text { al., } 2007\end{array}$ \\
\hline chr3:93,605,515-101,219,924 & $7,614,410$ & $\mathrm{q} 11.2-\mathrm{q} 12.3$ & Loss & 38 & 83.33333333 & $>0.05$ & N/A \\
\hline chr4:10,479,679-39,591,168 & $29,111,490$ & p16.1 - p14 & Loss & 62 & 83.33333333 & $>0.05$ & $\begin{array}{l}\text { Lockwood WW, et al., 2007; F.Y. Huang } \\
\text { et al.2005; Y.W. CHOI, et al., 2007; G } \\
\text { Ng, et al., } 2007\end{array}$ \\
\hline chr4:41,596,003-44,365,208 & $2,769,206$ & $\mathrm{p} 13$ & Loss & 10 & 83.33333333 & $>0.05$ & $\begin{array}{l}\text { Lockwood WW, et al., 2007; F.Y. Huang } \\
\text { et al.2005; G Ng, et al., } 2007\end{array}$ \\
\hline chr4:58,367,789-139,871,504 & $81,503,716$ & $\mathrm{q} 12-\mathrm{q} 31.1$ & Loss & 300 & 83.33333333 & $>0.05$ & $\begin{array}{c}\text { Lockwood WW, et al., 2007; G Ng, et } \\
\text { al., } 2007\end{array}$ \\
\hline $\begin{array}{l}\text { chr4:141,329,535- } \\
182,773,601\end{array}$ & $41,444,067$ & $\mathrm{q} 31.1-\mathrm{q} 34.3$ & Loss & 127 & 83.33333333 & $>0.05$ & $\begin{array}{l}\text { Lockwood WW, et al., 2007; Y.W. CHOI, } \\
\text { et al., 2007; G Ng, et al., } 2007\end{array}$ \\
\hline chr5:49,690,172-58,526,175 & $8,836,004$ & q11.1 - q11.2 & Loss & 35 & 83.33333333 & $>0.05$ & Connie P. Matthews, et al., 2000 \\
\hline $\operatorname{chr} 6: 44,372,753-58,614,061$ & $14,241,309$ & $\mathrm{p} 21.1-\mathrm{p} 11.2$ & Loss & 69 & 83.33333333 & $>0.05$ & $\begin{array}{l}\text { Connie P. Matthews, et al., 2000; Y.W. } \\
\text { CHOI, et al., } 2007\end{array}$ \\
\hline chr6:61,982,931-73,725,450 & $11,742,520$ & q11.1 - q13 & Loss & 16 & 83.33333333 & $>0.05$ & Connie P. Matthews, et al., 2000 \\
\hline $\operatorname{chr} 6: 75,067,155-105,246,238$ & $30,179,084$ & $\mathrm{q} 13-\mathrm{q} 21$ & Loss & 83 & 83.33333333 & $>0.05$ & Connie P. Matthews, et al., 2000 \\
\hline $\begin{array}{l}\text { chr6:112,398,800- } \\
148,256,165\end{array}$ & $35,857,366$ & $\mathrm{q} 21-\mathrm{q} 24.3$ & Loss & 143 & 83.33333333 & $>0.05$ & $\begin{array}{l}\text { Connie P. Matthews, et al., 2000; Y.W. } \\
\text { CHOI, et al., } 2007\end{array}$ \\
\hline $\operatorname{chr} 7: 76,075,269-97,170,202$ & $21,094,934$ & $\mathrm{q} 11.23-\mathrm{q} 21.3$ & Loss & 77 & 83.33333333 & $>0.05$ & N/A \\
\hline $\begin{array}{l}\operatorname{chr} 7: 105,159,121- \\
110,522,522\end{array}$ & $5,363,402$ & $\mathrm{q} 22.3-\mathrm{q} 31.1$ & Loss & 24 & 83.33333333 & $>0.05$ & $\mathrm{~N} / \mathrm{A}$ \\
\hline chr8:12,830,587-20,081,624 & $7,251,038$ & $\mathrm{p} 22-\mathrm{p} 21.3$ & Loss & 27 & 83.33333333 & $>0.05$ & $\begin{array}{c}\text { Lockwood WW, et al., 2007; G Ng, et } \\
\text { al., } 2007\end{array}$ \\
\hline chr8:75,336,800-85,510,468 & $10,173,669$ & $\mathrm{q} 21.11-\mathrm{q} 21.2$ & Loss & 27 & 83.33333333 & $>0.05$ & N/A \\
\hline $\begin{array}{l}\text { chr8:142,141,881- } \\
146,304,022\end{array}$ & $4,162,142$ & $\mathrm{q} 24.3$ & Gain & 92 & 83.33333333 & 0 & $\begin{array}{l}\text { Gopeshwar Narayan, et al., 2007; F.Y. } \\
\text { Huang et al.2005; Connie P. Matthews, et } \\
\text { al., 2000; Y.W. CHOI, et al., 2007; G Ng, } \\
\text { et al., } 2007\end{array}$ \\
\hline $\begin{array}{l}\text { chr9: } 128,223,213- \\
139,309,447\end{array}$ & $11,086,235$ & $\mathrm{q} 33.3-\mathrm{q} 34.3$ & Gain & 211 & 83.33333333 & 0.017 & $\begin{array}{c}\text { Lockwood WW, et al., 2007; Y.W. CHOI, } \\
\text { et al., } 2007\end{array}$ \\
\hline $\operatorname{chr} 11: 60,001-3,696,670$ & $3,636,670$ & $\mathrm{p} 15.5-\mathrm{p} 15.4$ & Gain & 85 & 83.33333333 & 0 & $\begin{array}{l}\text { Y.W. CHOI, et al., 2007; G Ng, et al., } \\
2007\end{array}$ \\
\hline $\operatorname{chr} 11: 20,658,997-31,795,373$ & $11,136,377$ & p15.1-p13 & Loss & 27 & 83.33333333 & $>0.05$ & F.Y. Huang et al.2005 \\
\hline chr11:65,627,563-67,839,841 & $2,212,279$ & q13.1 - q13.2 & Gain & 77 & 83.33333333 & 0.003 & Y.W. CHOI, et al., 2007 \\
\hline
\end{tabular}

(Continued) 


\begin{tabular}{|c|c|c|c|c|c|c|c|}
\hline Region & $\begin{array}{l}\text { Region } \\
\text { length }\end{array}$ & $\begin{array}{l}\text { Cytoband } \\
\text { location }\end{array}$ & Event & Genes & Frequency $\%$ & P-value & Reference \\
\hline $\operatorname{chr} 12: 38,766,104-42,827,028$ & $4,060,925$ & q12 & Loss & 12 & 83.33333333 & $>0.05$ & N/A \\
\hline chr13:45,697,630-55,761,181 & $10,063,552$ & q14.12 - q21.1 & Loss & 56 & 83.33333333 & $>0.05$ & $\begin{array}{l}\text { Lockwood WW, et al., 2007; G Ng, et al., } \\
\qquad 2007\end{array}$ \\
\hline chr14:23,251,874-24,898,017 & $1,646,144$ & $\mathrm{q} 11.2-\mathrm{q} 12$ & Gain & 64 & 83.33333333 & 0.001 & $\mathrm{~N} / \mathrm{A}$ \\
\hline chr16:60,001-3,153,334 & $3,093,334$ & $\mathrm{p} 13.3$ & Gain & 137 & 83.33333333 & 0.048 & Y.W. CHOI, et al., 2007 \\
\hline $\operatorname{chr} 16: 3,970,244-5,071,063$ & $1,100,820$ & $\mathrm{p} 13.3$ & Gain & 23 & 83.33333333 & 0.048 & Y.W. CHOI, et al., 2007 \\
\hline $\operatorname{chr} 16: 28,276,920-31,195,342$ & $2,918,423$ & $\mathrm{p} 11.2$ & Gain & 100 & 83.33333333 & 0.048 & Y.W. CHOI, et al., 2007 \\
\hline chr16:66,279,668-70,782,910 & $4,503,243$ & $\mathrm{q} 21-\mathrm{q} 22.1$ & Gain & 103 & 83.33333333 & 0.021 & $\begin{array}{l}\text { Lockwood WW, et al., 2007; Connie P. } \\
\text { Matthews, et al., } 2000\end{array}$ \\
\hline chr16:83,959,097-90,294,753 & $6,335,657$ & $\mathrm{q} 23.3-\mathrm{q} 24.3$ & Gain & 78 & 83.33333333 & 0.021 & $\begin{array}{c}\text { Lockwood WW, et al., 2007; Connie P. } \\
\text { Matthews, et al., 2000; Y.W. CHOI, et } \\
\text { al., } 2007\end{array}$ \\
\hline $\operatorname{chr} 17: 7,175,150-8,229,647$ & $1,054,498$ & $\mathrm{p} 13.1$ & Gain & 59 & 83.33333333 & 0.009 & N/A \\
\hline chr17:72,693,870-81,060,000 & $8,366,131$ & $\mathrm{q} 25.1-\mathrm{q} 25.3$ & Gain & 166 & 83.33333333 & $>0.05$ & $\begin{array}{c}\text { Lockwood WW, et al., 2007; Y.W. CHOI, } \\
\text { et al., } 2007\end{array}$ \\
\hline chr18:18,510,899-43,242,321 & $24,731,423$ & q11.1 - q12.3 & Loss & 70 & 83.33333333 & $>0.05$ & $\begin{array}{c}\text { Lockwood WW, et al., 2007; Y.W. CHOI, } \\
\text { et al., } 2007\end{array}$ \\
\hline chr18:62,450,433-71,009,737 & $8,559,305$ & $\mathrm{q} 22.1-\mathrm{q} 22.3$ & Loss & 11 & 83.33333333 & $>0.05$ & $\begin{array}{l}\text { Lockwood WW, et al., 2007; G Ng, et } \\
\text { al., } 2007\end{array}$ \\
\hline 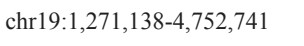 & $3,481,604$ & $\mathrm{p} 13.3$ & Gain & 105 & 83.33333333 & $>0.05$ & Y.W. CHOI, et al., 2007 \\
\hline chr19:12,747,550-14,740,086 & $1,992,537$ & p13.13 - p13.12 & Gain & 56 & 83.33333333 & $>0.05$ & Gopeshwar Narayan, et al., 2007 \\
\hline chr19:16,170,761-19,780,245 & $3,609,485$ & p13.12 - p13.11 & Gain & 103 & 83.33333333 & $>0.05$ & $\mathrm{~N} / \mathrm{A}$ \\
\hline chr19:45,216,651-51,316,691 & $6,100,041$ & q13.32 - q13.33 & Gain & 201 & 83.33333333 & $>0.05$ & Lockwood WW, et al., 2007 \\
\hline chr19:55,542,540-56,189,743 & 647,204 & $\mathrm{q} 13.42$ & Gain & 31 & 83.33333333 & $>0.05$ & N/A \\
\hline chr19:58,530,030-59,114,839 & 584,810 & $\mathrm{q} 13.43$ & Gain & 23 & 83.33333333 & $>0.05$ & N/A \\
\hline chr20:60,195,293-62,965,520 & $2,770,228$ & $\mathrm{q} 13.33$ & Gain & 63 & 83.33333333 & $>0.05$ & $\begin{array}{l}\text { Connie P. Matthews, et al., 2000; Y.W. } \\
\text { CHOI, et al., 2007; G Ng, et al., } 2007\end{array}$ \\
\hline chr21:14,417,523-32,339,619 & $17,922,097$ & q11.2 - q22.11 & Loss & 61 & 83.33333333 & $>0.05$ & N/A \\
\hline chrX:77,966,491-93,063,726 & $15,097,236$ & $\mathrm{q} 21.1-\mathrm{q} 21.32$ & Loss & 25 & 83.33333333 & $>0.05$ & Connie P. Matthews, et al., 2000 \\
\hline $\begin{array}{l}\text { chrX:120,138,580- } \\
127,769,411\end{array}$ & $7,630,832$ & $\mathrm{q} 24-\mathrm{q} 25$ & Loss & 10 & 83.33333333 & $>0.05$ & Connie P. Matthews, et al., 2000 \\
\hline $\begin{array}{l}\text { chrX:152,449,419- } \\
153,711,912\end{array}$ & $1,262,494$ & $\mathrm{q} 28$ & Gain & 47 & 83.33333333 & 0 & $\begin{array}{c}\text { Gopeshwar Narayan, et al., 2007; Connie } \\
\text { P. Matthews, et al., 2000 }\end{array}$ \\
\hline
\end{tabular}

$\mathrm{Mb}$ to $80 \mathrm{Mb}$ ), of which 11 have not been previously described in cervical cancer (Table 1). These differences in our results may be due to the different platforms of assay, different settings of analysis or the different cervical cancer cells. In these 50 commonly altered genomic regions, 3514 genes are included. Some of these, especially oncogenic or tumor suppressor genes, may be associated with the development of cervical cancer.

The gene tree clustering result suggested that during the development of cervical carcinoma, gene expression significantly changes, and cervical carcinoma can be distinguished from normal cervical epithelium tissue by clustering analysis of the gene expression profile. Since the cervical cancer cell lines were separate from primary cervical carcinoma and normal cervical epithelium tissue, we assumed that extended culturing of cervical cancer cell lines may also significantly alter their gene expression profiles.

The integrated analysis of genome-wide chromosomal copy number changes and gene expression profiling indicated that the identified CNVs could contribute to the expression of some but not all genes (Figure 1). This finding is consistent with a report by Vazquez-Mena et al. which used a different array platform to detect the correlation between CNVs and gene expression variation in cervical cancer cell lines. Other factors, such as epigenetic changes or transcription factors, may also contribute to variation of gene expression in cervical cancer [4]. Pathway analysis indicated that significant changes of some pathways, especially those involving the cell cycle, may be involved in the pathogenesis of cervical cancer. 

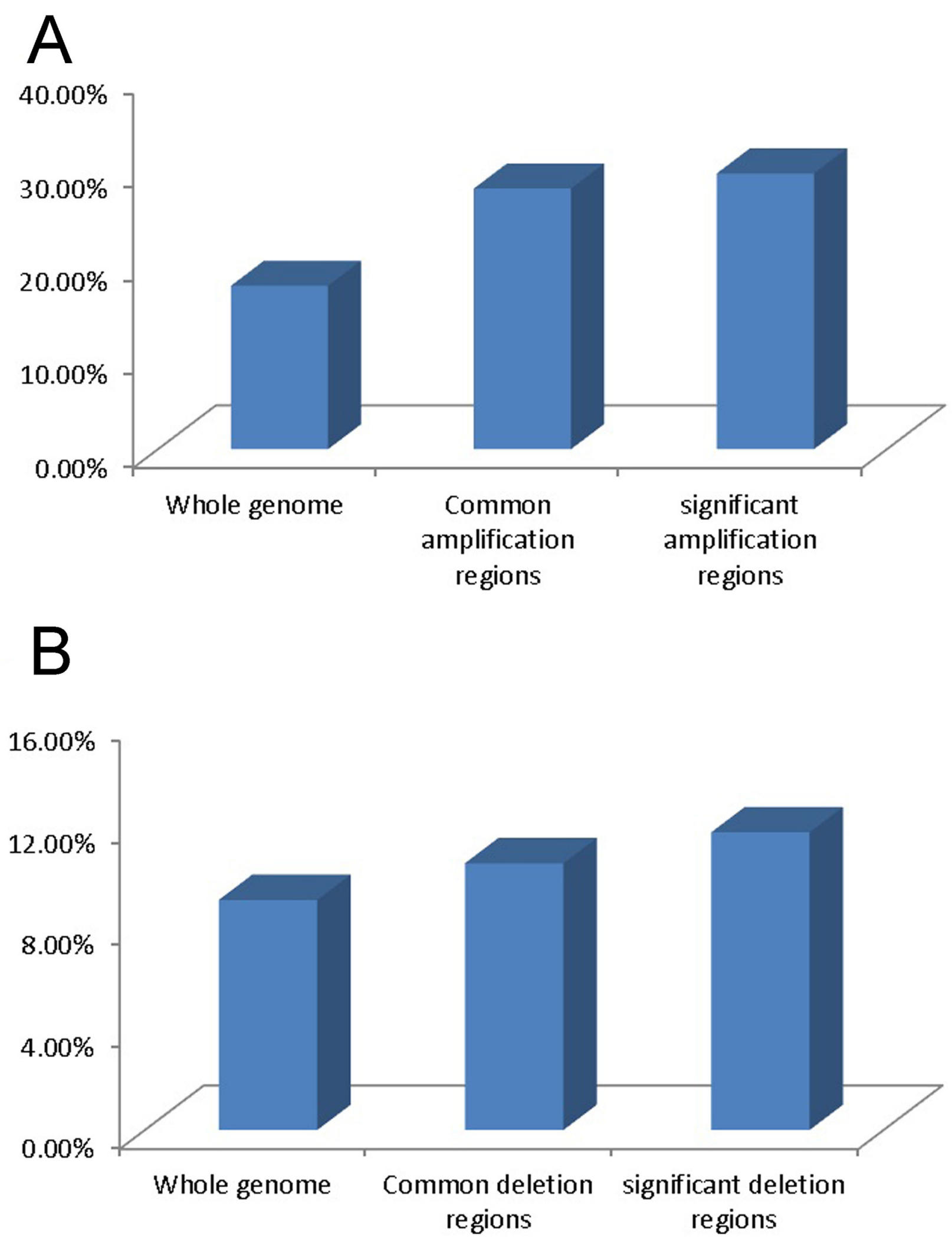

Figure 1: Gene expression variation in different genomic regions. (A) Percentage of up-regulated transcripts in whole genomic regions, common amplification regions and significant amplification regions. (B) Percentage of down-regulated transcripts in whole genomic regions, common deletion regions and significant deletion regions. 
$A B L 1$ and $p 53$, the two cell cycle pathway tumorrelated genes which were located in the significant genomic amplification regions, were found to be overexpressed at least 2-fold in cervical cancer. $A B L 1$, which was located in the chr9:128,223,213-139,309,447 genomic amplification region, plays a role in apoptosis. p53 is a well-known tumor suppressor gene, and an increase in $p 53$ levels plays a critical role in the induction of genes that results in cell cycle arrest [7], allowing repair of damaged DNA or activation of apoptotic pathways [8]. In cervical cancer with high risk of HPV-infection, the E6 protein from high-risk HPV can bind to tumor suppressor protein $\mathrm{p} 53$ for rapid degradation via a cellular ubiquitin ligase [9]. Other studies have indicated that $\mathrm{p} 53$ protein over-expression is not common or associated with survival in cervical carcinoma $[10,11]$. However, from our array CGH and gene expression array data, $p 53$ was located in the chr17:7,175,150-8,229,647 genomic amplification region and was over-expressed at the mRNA level (the median of the expression of $p 53$ in cervical cancer cell lines was 1.316 [from 0.82 to 3.38 ); the median of the expression of $p 53$ in normal cervical epithelium was 0.295 [from 0.153 to 0.485$]$ ). This suggests that gene dosage of p53 contributes to RNA over-expression in some cervical cancers.

Our results demonstrated that over-expression of transforming growth factor-beta 1 (TGF- $\beta 1$ ), a gene important in cell cycle pathways, may be due to a chromosomal CNV. TGF- $\beta 1$ was amplified and was also over-expressed ( $>2$ fold) in the cancer group compared with the normal group. TGF- $\beta 1$ is involved in many different critical processes, such as embryonic development, cellular maturation and differentiation, wound healing, immune regulation and inflammation. TGF- $\beta 1$ is a potent inhibitor of cell proliferation at the beginning of carcinogenesis $[12,13]$. When cells become resistant to TGF- $\beta 1$, tumor growth may be enhanced and metastasis promoted via immune evasion and angiogenesis. An increased expression of TGF- $\beta 1$ has been found in cervical cancer. Kirma et al. suggested that TGF- $\beta 1$ may be a factor in inducing over-expression of an oncogene, c-fms. Blocking c-fms has been demonstrated to result in increased apoptosis and decreased motility in cervical cancer [14].

Matrix metalloproteinases (MMPs) play an important role in the enhancement of tumor-induced angiogenesis. Our aCGH data showed that 9 MMP genes (MMP1, 3, 7, 8, 10, 12, 13, 20 and 27) located within 11q22 are amplified in Caski and SiHa cell lines, consistent with Lockwood's findings. Further analysis revealed that MMP14, 23B and 25 were located in significant genomic amplification regions and MMP1, 15, 17 and TIMP1 in all our 6 cervical cancer cell lines. This is consistent with previous work reporting over-expression of MMP12 and MMP15 [15-19].

In summary, we have identified several chromosomal CNV regions and demonstrated that chromosomal CNVs are a common phenomenon which can affect the level of RNA expression in cervical cancer. Pathway analysis for the aberrantly expressed genes
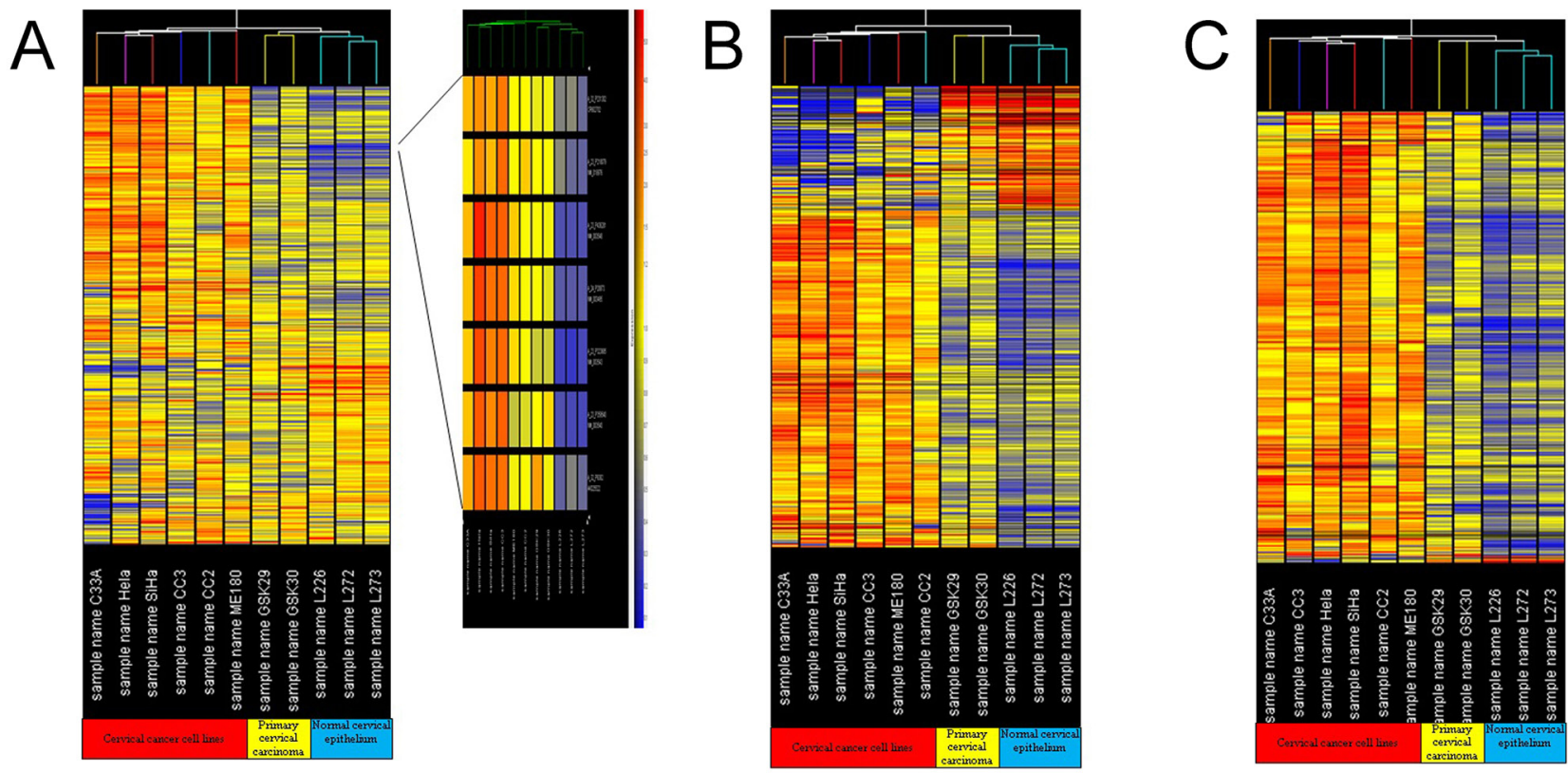

Figure 2: Gene tree clustering analysis. (A) Gene tree clustering analysis for the gene expression profiles of the cervical cancer cell lines, cervical carcinomas and normal cervical epithelium tissues; (B) gene tree clustering analysis for the aberrantly expressed genes in 50 common chromosomal CNV regions in cervical carcinoma; (C) gene tree clustering analysis for the aberrantly expressed genes in 15 significant chromosomal CNV regions in cervical carcinoma. 
A

CELLCYCLE

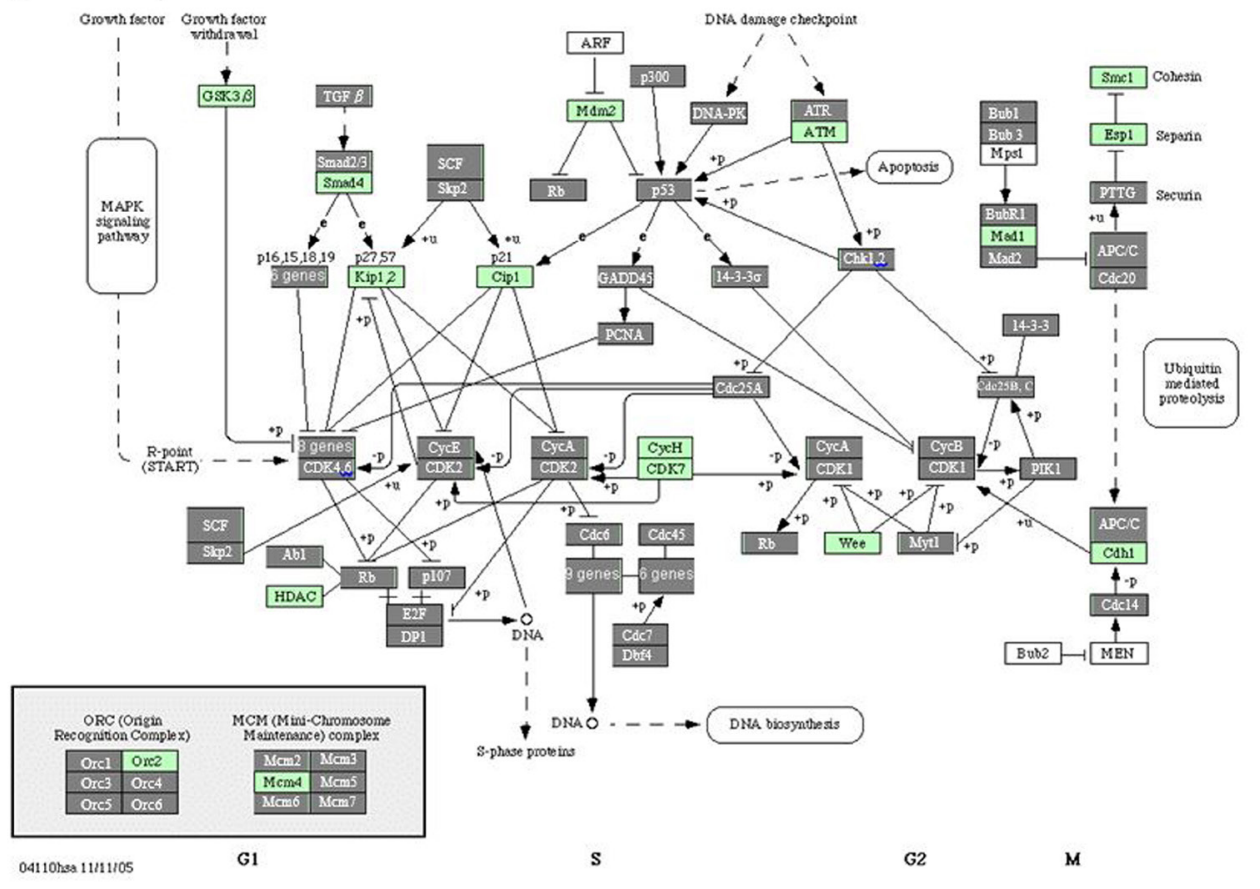

$\mathrm{B}$

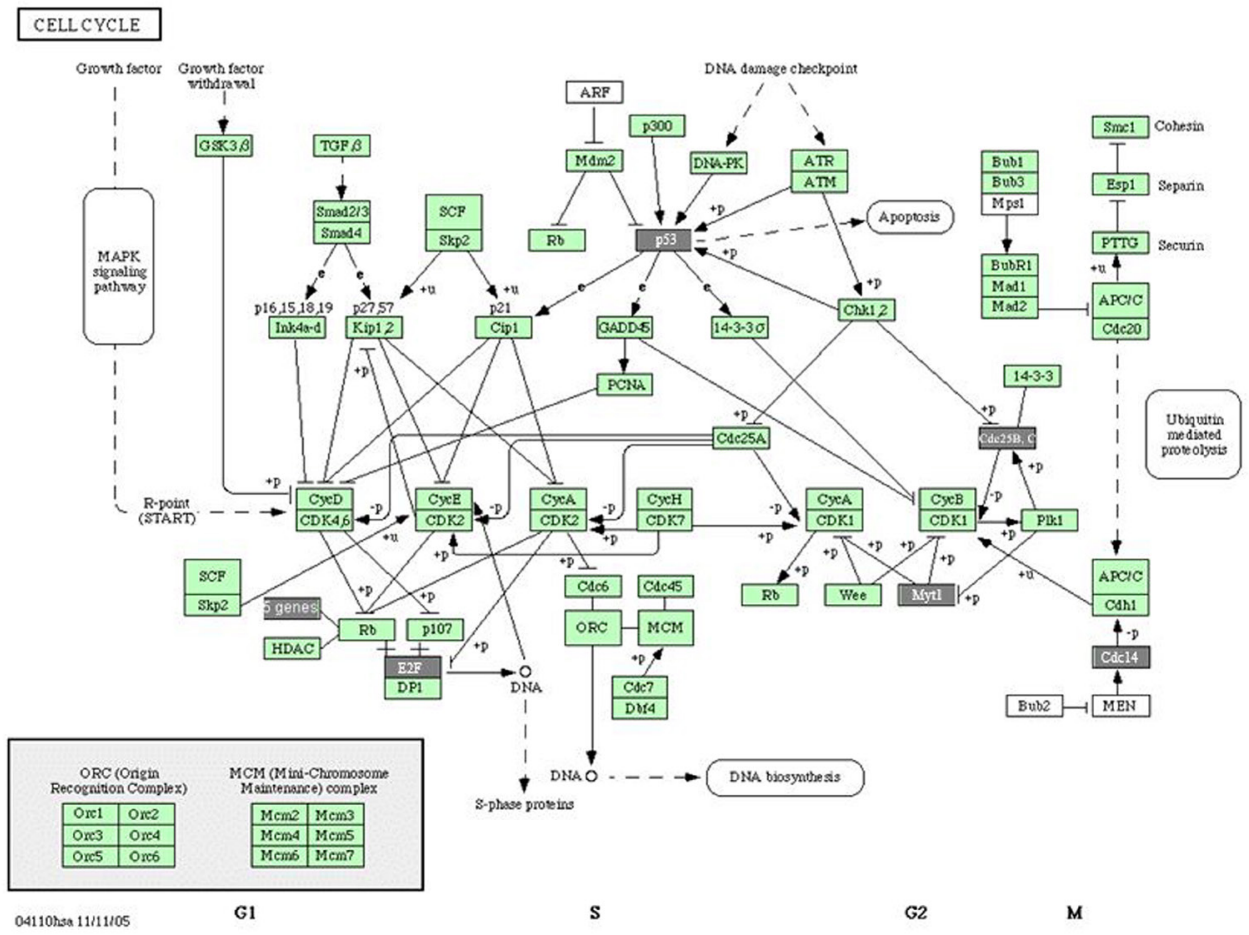

Figure 3: Cell cycle pathway analysis in cervical cancer. (A) Differently expressed genes involved in the cell cycle pathway in cervical carcinoma; (B) differently expressed genes within the significant chromosomal CNV regions involved in the cell cycle pathway in cervical carcinoma. Each rectangle represents one gene. The rectangle covered by gray color indicates that this gene is differently expressed in cervical carcinoma compared with normal cervix. 
Table 2: Pathway analysis for the differentially expressed genes in cervical cancer

\begin{tabular}{|c|c|c|}
\hline Pathway & $\begin{array}{l}\text { Number of genes with each } \\
\text { pathway }\end{array}$ & $\begin{array}{c}\text { Genelist vs pathway random } \\
\text { overlap p-value }\end{array}$ \\
\hline Cell cycle - Homo sapiens (human) & 111 & $1.15 \mathrm{E}-08$ \\
\hline Proteasome - Homo sapiens (human) & 27 & $2.66 \mathrm{E}-08$ \\
\hline One carbon pool by folate - Homo sapiens (human) & 22 & $5.11 \mathrm{E}-07$ \\
\hline Cell Communication - Homo sapiens (human) & 60 & $5.85 \mathrm{E}-07$ \\
\hline Pyrimidine metabolism - Homo sapiens (human) & 52 & $6.05 \mathrm{E}-06$ \\
\hline Purine metabolism - Homo sapiens (human) & 77 & $2.27 \mathrm{E}-05$ \\
\hline DNA polymerase - Homo sapiens (human) & 19 & $5.80 \mathrm{E}-05$ \\
\hline Arginine and proline metabolism - Homo sapiens (human) & 35 & 0.000103 \\
\hline Riboflavin metabolism - Homo sapiens (human) & 14 & 0.00036 \\
\hline gamma-Hexachlorocyclohexane degradation - Homo sapiens (human) & 17 & 0.000515 \\
\hline Hematopoietic cell lineage - Homo sapiens (human) & 45 & 0.000654 \\
\hline Valine, leucine and isoleucine biosynthesis - Homo sapiens (human) & 10 & 0.000804 \\
\hline Glycosphingolipid biosynthesis - ganglioseries - Homo sapiens (human) & 13 & 0.000894 \\
\hline Pathogenic Escherichia coli infection - EHEC - Homo sapiens (human) & 33 & 0.000941 \\
\hline Pathogenic Escherichia coli infection - EPEC - Homo sapiens (human) & 33 & 0.000941 \\
\hline Selenoamino acid metabolism - Homo sapiens (human) & 23 & 0.00105 \\
\hline Valine, leucine and isoleucine degradation - Homo sapiens (human) & 31 & 0.00139 \\
\hline Urea cycle and metabolism of amino groups - Homo sapiens (human) & 17 & 0.00172 \\
\hline ECM-receptor interaction - Homo sapiens (human) & 46 & 0.00179 \\
\hline Glycan structures - biosynthesis 2 - Homo sapiens (human) & 36 & 0.00261 \\
\hline 2,4-Dichlorobenzoate degradation - Homo sapiens (human) & 6 & 0.00264 \\
\hline Butanoate metabolism - Homo sapiens (human) & 24 & 0.00302 \\
\hline Lysine degradation - Homo sapiens (human) & 30 & 0.00449 \\
\hline Aminoacyl-tRNA biosynthesis - Homo sapiens (human) & 17 & 0.0047 \\
\hline Cell adhesion molecules (CAMs) - Homo sapiens (human) & 63 & 0.0062 \\
\hline Folate biosynthesis - Homo sapiens (human) & 22 & 0.00732 \\
\hline Ascorbate and aldarate metabolism - Homo sapiens (human) & 10 & 0.00742 \\
\hline Histidine metabolism - Homo sapiens (human) & 22 & 0.00918 \\
\hline Alkaloid biosynthesis II - Homo sapiens (human) & 14 & 0.0101 \\
\hline Limonene and pinene degradation - Homo sapiens (human) & 18 & 0.0102 \\
\hline Focal adhesion - Homo sapiens (human) & 91 & 0.0108 \\
\hline Methionine metabolism - Homo sapiens (human) & 11 & 0.0112 \\
\hline Oxidative phosphorylation - Homo sapiens (human) & 49 & 0.012 \\
\hline Citrate cycle (TCA cycle) - Homo sapiens (human) & 16 & 0.0153 \\
\hline Glycosaminoglycan degradation - Homo sapiens (human) & 11 & 0.016 \\
\hline Nitrobenzene degradation - Homo sapiens (human) & 10 & 0.0165 \\
\hline Olfactory transduction - Homo sapiens (human) & 17 & 0.0179 \\
\hline Glycolysis Gluconeogenesis - Homo sapiens (human) & 31 & 0.0197 \\
\hline Ethylbenzene degradation - Homo sapiens (human) & 11 & 0.0221 \\
\hline Ubiquitin mediated proteolysis - Homo sapiens (human) & 26 & 0.0236 \\
\hline
\end{tabular}

(Continued) 


\begin{tabular}{lcc}
\hline Pathway & $\begin{array}{c}\text { Number of genes with each } \\
\text { pathway }\end{array}$ & $\begin{array}{c}\text { Genelist vs pathway random } \\
\text { overlap p-value }\end{array}$ \\
\hline Glyoxylate and dicarboxylate metabolism - Homo sapiens (human) & 9 & 0.0243 \\
Arachidonic acid metabolism - Homo sapiens (human) & 25 & 0.0306 \\
N-Glycan biosynthesis - Homo sapiens (human) & 21 & 0.033 \\
Chondroitin sulfate biosynthesis - Homo sapiens (human) & 7 & 0.037 \\
Linoleic acid metabolism - Homo sapiens (human) & 17 & 0.0415 \\
Pentose phosphate pathway - Homo sapiens (human) & 14 & 0.0465 \\
Apoptosis - Homo sapiens (human) & 38 & 0.0467 \\
Protein export - Homo sapiens (human) & 8 & 0.0498 \\
\hline
\end{tabular}

Table 3: Pathway analysis for the differentially expressed genes within the 15 significant chromosome CNV regions in cervical cancer

\begin{tabular}{|c|c|c|}
\hline Pathway & $\begin{array}{l}\text { Number of common genes } \\
\text { with each pathway }\end{array}$ & $\begin{array}{c}\text { Genelist vs pathway random } \\
\text { overlap p-value }\end{array}$ \\
\hline Cell cycle - Homo sapiens (human) & 11 & 0.000622 \\
\hline Purine metabolism - Homo sapiens (human) & 7 & 0.0126 \\
\hline Axon guidance - Homo sapiens (human) & 6 & 0.0365 \\
\hline Insulin signaling pathway - Homo sapiens (human) & 6 & 0.0386 \\
\hline Selenoamino acid metabolism - Homo sapiens (human) & 5 & 0.000365 \\
\hline Tyrosine metabolism - Homo sapiens (human) & 5 & 0.00382 \\
\hline Glycerophospholipid metabolism - Homo sapiens (human) & 5 & 0.00419 \\
\hline Tryptophan metabolism - Homo sapiens (human) & 5 & 0.0137 \\
\hline Nitrobenzene degradation - Homo sapiens (human) & 4 & 0.000101 \\
\hline Aminophosphonate metabolism - Homo sapiens (human) & 4 & 0.000199 \\
\hline Histidine metabolism - Homo sapiens (human) & 4 & 0.00452 \\
\hline Androgen and estrogen metabolism - Homo sapiens (human) & 4 & 0.00747 \\
\hline Glycan structures - biosynthesis 2 - Homo sapiens (human) & 4 & 0.0281 \\
\hline Sulfur metabolism - Homo sapiens (human) & 3 & 0.000724 \\
\hline Ethylbenzene degradation - Homo sapiens (human) & 3 & 0.00309 \\
\hline RNA polymerase - Homo sapiens (human) & 3 & 0.00781 \\
\hline $\begin{array}{l}\text { 1- and 2-Methylnaphthalene degradation - Homo sapiens } \\
\text { (human) }\end{array}$ & 3 & 0.0136 \\
\hline Benzoate degradation via CoA ligation - Homo sapiens (human) & 3 & 0.0153 \\
\hline Limonene and pinene degradation - Homo sapiens (human) & 3 & 0.0153 \\
\hline Pyruvate metabolism - Homo sapiens (human) & 3 & 0.0362 \\
\hline Bisphenol A degradation - Homo sapiens (human) & 2 & 0.0233 \\
\hline $\begin{array}{l}\text { Glycosphingolipid biosynthesis - ganglioseries - Homo sapiens } \\
\text { (human) }\end{array}$ & 2 & 0.03 \\
\hline $\begin{array}{l}\text { Dentatorubropallidoluysian atrophy (DRPLA) - Homo sapiens } \\
\text { (human) }\end{array}$ & 2 & 0.0323 \\
\hline Parkinson's disease - Homo sapiens (human) & 2 & 0.0323 \\
\hline
\end{tabular}


suggested that significant changes of some pathways, especially those involving the cell cycle, may contribute to the pathogenesis of cervical cancer. This study provided some clinical significance for us to have a better understanding of cervical cancer pathogenesis.

\section{MATERIALS AND METHODS}

\section{Cervical cancer cell lines and specimens}

Six human cervical cancer cell lines (HeLa, SiHa, C33A, ME180, CC2 and CC3) were used for aCGH and gene expression array. Five clinical specimens, including three normal cervical tissues and two cervical carcinoma specimens (FIGO stage: $\mathrm{II}_{\mathrm{A}}$ ), were collected from patients (aged between 36-42 years old) at the Department of Obstetrics and Gynaecology at the Prince of Wales Hospital in Hong Kong from January 2014 to December 2014. Informed consent was obtained from all participating subjects, and Institutional Review Board approval was obtained.

\section{Tissue micro-dissection}

Micro-dissection was used as described in our previous study [5]. Briefly, the tissue specimens were frozen in OCT cryomoulds (SAKURA, Japan), sectioned $(8 \mu \mathrm{m})$ by a cryostat at $-20^{\circ} \mathrm{C}$ (Leica Corp., CRYOCUT 1800 ), and then mounted onto glass slides (SAIL BRAND, Cat No 7105) at room temperature. Sections were stained by $0.1 \%$ methyl green (Sigma) and micro-dissected using a sterile surgical blade (AESCULAP) and collected immediately for further experiments.

\section{Microarray comparative genomic hybridization analysis}

Microarray comparative genomic hybridization using Human Genome CGH Microarray Kit $(4 \mathrm{x} 44 \mathrm{~K})$ (Agilent Technologies, Santa Clara, CA, USA) was used for identifying chromosomal CNV of 6 cervical cancer cell lines and 3 normal cervical samples as per the manufacturer's protocol. Briefly, genomic DNA of cervical cancer cell lines was extracted using a DNeasy Blood \& Tissue Kit (QIAGEN, Cat No. 69506). $1 \mu \mathrm{g}$ genomic DNA of test sample and $1 \mu \mathrm{g}$ human sex-matched control DNA as a reference sample (Promega G1521A; Lot no. 20929604) were digested using Alu I and Rsa I. This was followed by fluorescent labeling, clean-up of labeled genomic DNA, microarray hybridization and scanning. The data were extracted using the Agilent Feature Extraction (FE) v11.0 program. After calculating the background signal, non-uniform signal and the average raw signal on each probe, the resulting data files were generated and transferred to the bioinformatics software,
Nexus Copy Number version 6.1 (BioDiscovery, Inc., El Segundo, CA, USA) for analysis [6].

\section{Gene expression analysis}

The Whole Human Genome Microarray Kit, 4x44K (G4112F) was used to probe gene expression in 6 cervical cancer cell lines, 2 cervical carcinomas and 3 normal cervical epithelium tissue samples. The resulting data files were generated and transferred to GeneSpring GX version 11.5 (Agilent Technologies, Santa Clara, CA, USA) for further analysis.

\section{Reverse transcription PCR (RT-RCR)}

RT-RCR was peformed for the identified gain and loss genes both on expression level and on genome level, normalized by GAPDH and B-globin (Forward: 5'-GAAGAGCCAAGGACAGGTAC-3', Reverse: 5'-CAACTTCATCCACGTTCACC-3'), B2M (Forward:5'TGCTGTCTCCATGTTTGATGTATCT-3'; Reverse:5'TCTCTGCTCCCCACCTCTAAGT-3'), respectively.

\section{Statistical analysis}

For the Microarray Comparative Genomic Hybridization Analysis, 0.37 was used as the cut off value for amplification or 0.5 for deletion of a single probe. Putative chromosome copy number changes were defined by intervals of three or more adjacent probes with $\log _{2}$ ratios suggestive of a deletion or duplication when compared with the $\log _{2}$ ratios of adjacent probes. The $\mathrm{p}$ value for significant difference was set to less than 0.05 to reduce the false discovery rate (FDR). For Gene Expression Analysis, the cut-off value defining an aberrant change of gene expression was set at 2 fold for data analysis. Pathway analysis for the gene expression data was performed by GeneSpring GX version 11.5, and the pathway was downloaded from the KEGG database (ftp://ftp.genome.jp/pub/kegg/).

\section{Abbreviations}

CNV Copy number variations

HPV Human papillomavirus

CGH Comparative genomic hybridization

TGF- $\beta 1$ Transforming growth factor-beta 1

MMPs Matrix metalloproteinases

FDR False discovery rate

\section{Author contributions}

T.T., Y.S. and T.Y. designed the research. Y.D. and Y.S. performed the experiments and wrote the manuscript. C.C.W., H.K.W., T.K.H.C., L.X.H. and K.W.C. revised the manuscript. All authors reviewed the manuscript. 


\section{CONFLICTS OF INTEREST}

None of the authors of this manuscript report conflicts of interest regarding the performance of this study or its publication.

\section{FUNDING}

This study was supported by Basic Research for 2015 Shenzhen Municipal Science and Technology Programme (JCY20150630165236963), Shenzhen Science and Technology Innovation Project (JCYJ20140414114853648), the Innovation and Technology Fund of the Hong Kong Special Administrative Region, China (Ref No. UIM/283) and grants from the National Natural Science Foundation of China (grant No. 81660150 and 81372750).

\section{REFERENCES}

1. Bodelon C, Vinokurova S, Sampson JN, den Boon JA, Walker JL, Horswill MA, Korthauer K, Schiffman M, Sherman ME, Zuna RE, Mitchell J, Zhang X, Boland JF, et al. Chromosomal copy number alterations and HPV integration in cervical precancer and invasive cancer. Carcinogenesis. 2016; 37: 188-96.

2. Lockwood WW, Coe BP, Williams AC, MacAulay C, Lam WL. Whole genome tiling path array CGH analysis of segmental copy number alterations in cervical cancer cell lines. Int J Cancer. 2007; 120: 436-43.

3. Lando M, Holden M, Bergersen LC, Svendsrud DH, Stokke T, Sundfor K, Glad IK, Kristensen GB, Lyng H. Gene dosage, expression, and ontology analysis identifies driver genes in the carcinogenesis and chemoradioresistance of cervical cancer. PLoS Genet. 2009; 5: e1000719.

4. Vazquez-Mena O, Medina-Martinez I, Juarez-Torres E, Barron V, Espinosa A, Villegas-Sepulveda N, Gómez-Laguna L, Nieto-Martínez K, Orozco L, Roman-Basaure E, Muñoz Cortez S, Borges Ibañez M, Venegas-Vega C, et al. Amplified genes may be overexpressed, unchanged, or downregulated in cervical cancer cell lines. PLoS One. 2012; 7: e32667.

5. Tang T, Wong HK, Gu W, Yu MY, To KF, Wang CC, Wong YF, Cheung TH, Chung TK, Choy KW. MicroRNA-182 plays an onco-miRNA role in cervical cancer. Gynecol Oncol. 2013; 129: 199-208.

6. Tusher VG, Tibshirani R, Chu G. Significance analysis of microarrays applied to the ionizing radiation response. Proc Natl Acad Sci U S A. 2001; 98: 10515.

7. Orth JD, Loewer A, Lahav G, Mitchison TJ. Prolonged mitotic arrest triggers partial activation of apoptosis, resulting in DNA damage and p53 induction. Mol Biol Cell. 2012; 23: 567-76.

8. Wang X, Simpson ER, Brown KA. p53: Protection against tumor growth beyond effects on cell cycle and apoptosis. Cancer Res. 2015; 75: 5001-07.

9. Hiller T, Stubenrauch F, Iftner T. Isolation and functional analysis of five HPVE6 variants with respect to p53 degradation. J Med Virol. 2008; 80: 478-83.

10. Tommasino M, Accardi R, Caldeira S, Dong W, Malanchi I, Smet A, Zehbe I. The role of TP53 in cervical carcinogenesis. Hum Mutat. 2003; 21: 307-12.

11. Koyamatsu Y, Yokoyama M, Nakao Y, Fukuda K, Saito T, Matsukuma K, Iwasaka T.. A comparative analysis of human papillomavirus types 16 and 18 and expression of p53 gene and Ki-67 in cervical, vaginal, and vulvar carcinomas. Gynecol Oncol. 2003; 90: 547-51.

12. Mantel PY, Schmidt-Weber CB. Transforming growth factor-beta: recent advances on its role in immune tolerance. Methods Mol Biol. 2011; 677: 303-38.

13. Kloth JN, Fleuren GJ, Oosting J, de Menezes RX, Eilers $\mathrm{PH}$, Kenter GG, Gorter A. Substantial changes in gene expression of Wnt, MAPK and TNFalpha pathways induced by TGF-beta1 in cervical cancer cell lines. Carcinogenesis. 2005; 26: 1493-502.

14. Kirma N, Hammes LS, Liu YG, Nair HB, Valente PT, Kumar S, Flowers LC, Tekmal RR. Elevated expression of the oncogene c-fms and its ligand, the macrophage colony-stimulating factor-1, in cervical cancer and the role of transforming growth factor-beta1 in inducing c-fms expression. Cancer Res. 2007; 67: 1918-26.

15. Luo CF, Zhu RQ, Wang H, Lu YL. Expression of COX-2 and MMP-9 in cervical carcinoma and their clinical significance. Zhonghua Zhong Liu Za Zhi. 2007; 29: 526-30.

16. Rauvala M, Aglund K, Puistola U, TurpeenniemiHujanen T, Horvath G, Willen R, Stendahl U. Matrix metalloproteinases-2 and -9 in cervical cancer: different roles in tumor progression. Int J Gynecol Cancer. 2006; 16: 1297-302.

17. Abraham R, Schafer J, Rothe M, Bange J, Knyazev P, Ullrich A. Identification of MMP-15 as an anti-apoptotic factor in cancer cells. J Biol Chem. 2005; 280: 34123-32.

18. Vazquez-Ortiz G, Pina-Sanchez P, Vazquez K, Duenas A, Taja L, Mendoza P, Garcia JA, Salcedo M. Overexpression of cathepsin F, matrix metalloproteinases 11 and 12 in cervical cancer. BMC Cancer. 2005; 5: 68.

19. Nasr M, Ayyad SB, El-Lamie IK, Mikhail MY. Expression of matrix metalloproteinase-2 in preinvasive and invasive carcinoma of the uterine cervix. Eur J Gynaecol Oncol. 2005; 26: 199-202. 